\title{
COMPOSIÇÃO FLORÍSTICA E ESTRUTURA DE FLORESTA EM VÁRZEA ALTA ESTUARINA AMAZÔNICA
}

\author{
José Antonio Leite de Queiroz*, Silas Mochiutti**, Sebastião do Amaral Machado***, \\ Franklin Galvão**** \\ * Eng. Florestal, M.Sc., EMBRAPA-Amapá - leite.queiroz@terra.com.br \\ ** Eng. Agrônomo, M.Sc., EMBRAPA-Amapá - silas@uol.com.br \\ *** Eng. Florestal, Dr., Depto. de Ciências Florestais, UFPR - sammac@floresta.ufpr.br \\ **** Eng. Florestal, Dr., Depto. de Ciências Florestais, UFPR - fgalvao@floresta.ufpr.br \\ Recebido para publicação: 18/12/2003 - Aceito para publicação: 24/03/2005
}

\begin{abstract}
Resumo
Composição floristica e estrutura de floresta em várzea alta estuarina amazônica. O presente estudo foi desenvolvido objetivando avaliar a composição florística e a estrutura de floresta de várzea alta do estuário amazônico. Uma parcela amostral de 1,0 ha, dividida em subparcelas de 20 x $50 \mathrm{~m}$, foi instalada no Bailique/AP, furo do Mazagão/AP e rio Maniva/PA, e medidos os indivíduos com DAP $\geq 5 \mathrm{~cm}$. Foram identificados 69 espécies e 60 gêneros de 29 famílias, sendo que 21 espécies foram comuns aos três locais e 24 a apenas um dos três locais. As famílias mais abundantes foram: Arecaceae com 416 plantas/ha (50,4\%), com a espécie Euterpe oleracea Mart., 207 plantas/ha $(25,1 \%)$ e Astrocaryum murumuru Mart. 160 plantas/ha (19,4\%); Caesalpiniaceae com 95 plantas/ha $(11,5 \%)$ com a espécie Mora paraensis Ducke, 82 plantas/ha $(9,9 \%)$ e família Mimosaceae com 83 plantas/ha (10,0\%), com a espécie Pentaclethra macroloba (Willd.) Kuntze 56 plantas/ha (6,7\%). Para dominância absoluta: 30,07; 34,18; e $36,56 \mathrm{~m}^{2} /$ ha. Diversidade de espécies: 2,$31 ; 2,67$ e 2,84. Quociente de mistura: 1:18, 1:18 e 1:20 no Bailique, Mazagão e Maniva, respectivamente.

Palavras-chave: Amazônia; fitossociologia; espécies florestais.
\end{abstract}

\begin{abstract}
Floristic composition and forest's structure in Amazon estuarine high floodplain. The present study was developed aiming to evaluate the floristic composition and the structure of high floodplain forest in the Amazon estuary. One sample plot of one hectare divided in sub-plots of $20 \times 50 \mathrm{~m}$, were installed in Bailique/AP, furo do Mazagão/AP and rio Maniva/PA, for measurement of all trees with $\mathrm{DBH} \geq 5 \mathrm{~cm}$. It was identified 69 species and 60 genera of 29 families, with 21 species common to the three areas and 24 only at one of the three areas. The families most abundant were: Arecaceae with 416 plants/ha $(50,4 \%)$ with the specie Euterpe oleracea Mart. 207 plants/ha $(25,1 \%)$ and Astrocaryum murumuru Mart. 160 plants/ha (19,4\%), Caesalpiniaceae with 95 plants/ha (11,5\%) with the specie Mora paraensis Ducke 82 plants/ha $(9,9 \%)$, and Mimosaceae family with 83 plants/ha $(10,0 \%)$, with Pentaclethra macroloba (Willd.) Kuntze 56 plants/ha (6,7\%). Absolute dominance were: 30,$07 ; 34,18$; and $36,56 \mathrm{~m}^{2} / \mathrm{ha}$. Species diversity: 2,$31 ; 2,67$ and 2,84. Mixture quotients: 1:18, $1: 18$ and 1:20 for Bailique, Mazagão and Maniva, respectively.

Keywords: amazonian; phytossociology; wood species.
\end{abstract}

\section{INTRODUÇÃO}

O Estado do Amapá, situado no extremo Norte do Brasil, possui uma área de 14,3 milhões de hectares, dos quais mais de $3 \%$ são compostos por florestas de várzea estuarina, rica em espécies madeiráveis e não madeiráveis. Entre as não madeiráveis destaca-se o açaizeiro (Euterpe oleraceae Mart.), uma Arecaceae de porte elegante, produtora de frutos comestíveis, da qual extrai-se o palmito, produto utilizado na indústria de conservas para comercialização no Brasil e no exterior.

Rios e lagos da hiléia são muitas vezes acompanhados por faixas de terrenos baixos, sujeitos a inundação durante um determinado período de cada ano. Essas terras baixas são chamadas várzeas e a floresta que as cobre chama-se "mata de várzea", em contraste com a "mata da terra-firme". As terras 
baixas da zona costeira da hiléia e do grande estuário amazônico são inundadas pela repercussão das marés atlânticas. A "mata" dos lugares mais baixos, diariamente inundados, aproxima-se do igapó e a dos lugares mais altos, inundados somente pelas marés grandes, assemelha-se à da várzea de outras partes da região (Ducke e Black, 1954).

O regime de inundação, as diferenças no teor de sedimentos na água, a distância do ponto de origem dos sedimentos e das várzeas das margens dos respectivos rios, a intensidade da inundação e a influência da maré e da água do mar determinam desigualdades significativas no revestimento florístico, na formação do solo, nas características físicas e químicas e na potencialidade agropecuária das áreas inundáveis pelos rios de águas barrentas (Lima \& Tourinho, 1994). As utilizações desse ambiente estão centradas no extrativismo vegetal, principalmente açaí (fruto e palmito), seringa, andiroba, madeira e pecuária extensiva (ZEE/AP, 2000).

A produção de frutos e a de palmito de açaí depende da relação entre o número de touceiras de açaizeiros por hectare, demais palmeiras e espécies lenhosas (Queiroz \& Mochiutti, 2002). Considerando que o número total de árvores por hectare raramente ultrapassa mil, o aumento na população de açaizeiros implicará na diminuição da população de dicotiledôneas.

Segundo Anderson et al. (1985) o açaizeiro é uma das plantas mais abundantes e freqüentes nas áreas de várzea, constituindo-se na espécie nativa de maior importância econômica para a região do estuário amazônico, podendo atingir até 25\% da população vegetal das áreas de várzea. Nogueira (1999), em Igarapé Miri/PA, em áreas de vegetação original pouco alterada, encontrou populações de açaizeiros até cinco vezes superiores às encontradas por outros estudiosos. Segundo o autor, isso decorreu da intensa exploração efetuada pelos habitantes locais, que eliminaram quase que por completo as espécies consideradas de baixo valor comercial, de ocorrência natural nas áreas de várzea.

Segundo Dubois et al. (1996), as comunidades que começaram a manejar seus açaizais têm a tendência de manter em pé os açaizeiros e eliminar as plantas que fazem sombra aos açaizeiros.

O presente trabalho foi realizado com o objetivo de analisar a composição florística e a estrutura de floresta em área de várzea alta estuarina Amazônica, em local de coleta de frutos de açaí e extração de palmito, visando fornecer subsídios para o estabelecimento de projetos de manejo de açaizais e a exploração em regime de baixo impacto.

\section{MATERIAL E MÉTODOS}

A localização das áreas foi feita com a colaboração de membros da equipe responsável pela realização do Zoneamento Ecológico Econômico do Estado do Amapá, com base nas informações oriundas da interpretação de imagens do satélite Landsat (Figura 1). Inicialmente escolheu-se as localidades de Mazagão/AP, margem esquerda do rio Amazonas; Ilha do Pará, Afuá/PA, margem direita do rio Amazonas e Vila Progresso, Arquipélago do Bailique, Macapá/AP, constituído por ilhas fluviais, na foz do rio Amazonas. 


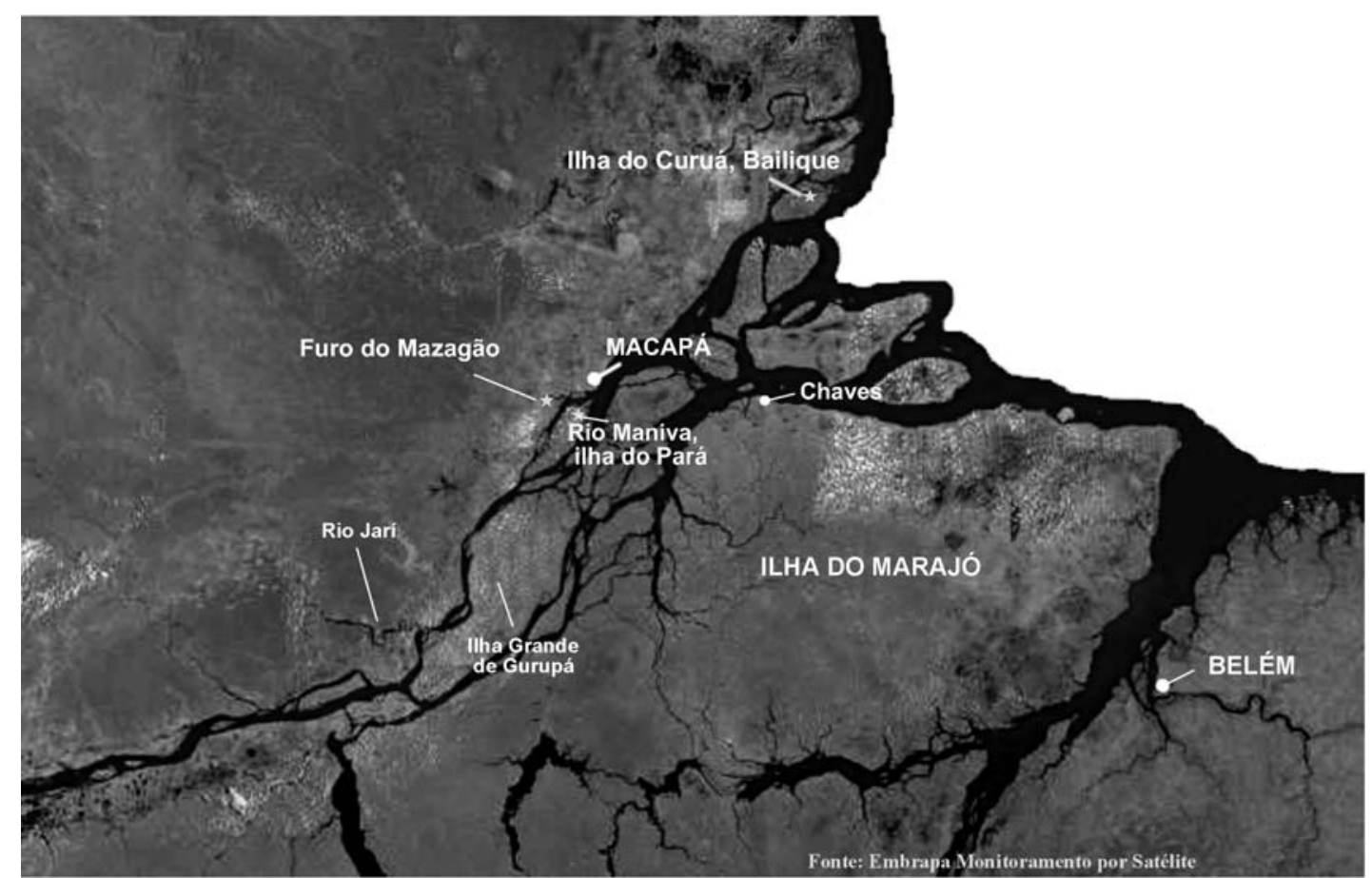

Figura 1. Localização das áreas de estudo no estuário amazônico.

Figure 1. Localization of study areas in the Amazon estuary.

Foram escolhidas áreas no Bailique, no furo do Mazagão e no rio Maniva, por se tratar de comunidades cuja utilização principal dos recursos da floresta seja a coleta regular de frutos de açaí e as extrações de palmito e de madeira serem feitas visando manter ou melhorar a produção de frutos e facilitar a coleta dos cachos de açaí.

A área do Bailique foi escolhida por situar-se na foz do rio Amazonas, local dos mais distantes em relação ao fornecimento de frutos de açaí para o consumo em Macapá e em Santana. A área de Mazagão, além de receber o mesmo tipo de manejo e estar localizada próxima de Macapá e de Santana, por situar-se na margem esquerda do rio Amazonas. A área do rio Maniva, por situar-se na margem direita do rio Amazonas. Portanto, áreas com recursos vegetais equivalentes, mas com potencialidades econômicas diferentes, em razão das diferentes distâncias a que se encontram dos principais centros consumidores de frutos de açaí, no estado do Amapá.

Para a instalação das amostras, realizou-se levantamento de informações junto aos moradores locais sobre a ocorrência e localização de áreas de floresta que se aproximassem da condição natural, isto é, que a última extração de madeiras tivesse ocorrido de forma seletiva, com o mínimo de impacto e que a última intervenção tivesse ocorrido, pelo menos, há 10 anos.

$\mathrm{O}$ estudo foi feito em locais com predomínio de várzea alta $(>95 \%)$, por ser o preferido para a coleta de frutos de açaí e para a exploração do palmito. Em cada um dos três locais estudados instalou-se uma amostra de um hectare, com dez subparcelas de 20 x $50 \mathrm{~m}$. Considerou-se várzea alta os locais que no período de marés grandes, a água invade o solo e sai logo depois, deixando-o em boas condições de drenagem. Estes períodos ocorrem durante os meses mais chuvosos, que são os de dezembro/janeiro a junho/julho. Nos demais meses o solo da várzea alta fica completamente seco.

Para o estudo da vegetação foram considerados todos os indivíduos com DAP (Diâmetro à Altura do Peito) maior ou igual a 5,0 cm. Após a identificação da árvore pelo nome popular, mediu-se o diâmetro do fuste a 1,30 m de altura, com fita métrica. Para os açaizeiros, contou-se cada touceira como apenas um indivíduo, visto que os estipes têm origem de uma mesma semente.

A identificação das árvores foi feita por profissionais experientes e as espécies sobre as quais se tinham dúvidas foram levadas para o Herbário Amapaense - HAMAB, do Instituto de Pesquisas 
Científicas e Tecnológicas do Estado do Amapá - IEPA, para comparação com exsicatas dos referidos materiais. Algumas espécies, por não apresentarem flores nas coletas, não foram identificadas.

A composição florística foi analisada através da comparação da distribuição dos indivíduos, agrupados em famílias e espécies.

A densidade foi calculada de acordo com Curtis e McIntosh (1950) e Mueller-Dombois e Ellenberg (1974) e o valor de importância com Curtis (1959). A freqüência absoluta foi obtida pela porcentagem das parcelas em que a espécie ocorreu e a relativa da relação entre freqüência absoluta de uma determinada espécie sobre a das demais, em porcentagem. A dominância absoluta foi obtida pela soma das áreas transversais dos indivíduos de uma mesma espécie para cada área e a relativa da participação, em porcentagem, de cada espécie em relação à área basal total. Foi considerada a área transversal medida a $1,30 \mathrm{~m}$ do solo.

O grau de agregação das espécies foi obtido de acordo com o índice de dispersão de Mc Guinnes (IGA), citado por Barros e Machado (1984), onde IGA $>1$, indica uma tendência da espécie ao agrupamento, IGA $>2$, sugere que a espécie apresenta um padrão de distribuição contagiosa, IGA $=1$, indica que a espécie apresenta tendência de distribuição aleatória e IGA $<1$, sugere que a espécie tem uma distribuição uniforme.

O índice de diversidade de Shannon-Weaver (H'), a equabilidade (J) e o quociente de mistura de Jentsch, foram obtidos de acordo com citação feita por Rabelo (1999).

\section{RESULTADOS E DISCUSSÃO}

Ao todo foram encontrados 69 espécies e 60 gêneros de 29 famílias, sendo que 21 espécies foram comuns ao Bailique, ao furo do Mazagão e ao rio Maniva, 24 espécies foram encontradas em apenas um desses locais e 24 foram encontradas em dois desses locais (Tabela 1). No furo do Mazagão foram encontrados 897 indivíduos com DAP $\geq 5,0 \mathrm{~cm}$ distribuídos em 25 famílias e 49 espécies. No rio Maniva, 860 indivíduos distribuídos em 24 famílias e 49 espécies. No Bailique, 721 indivíduos distribuídos em 18 famílias e 37 espécies.

De acordo com a expressão gráfica externada pela curva espécie/área, pode-se considerar que a amostragem foi bem representativa para os três locais estudados, visto que houve estabilização no surgimento de espécies novas entre a oitava e a nona subparcela (Figura 2).
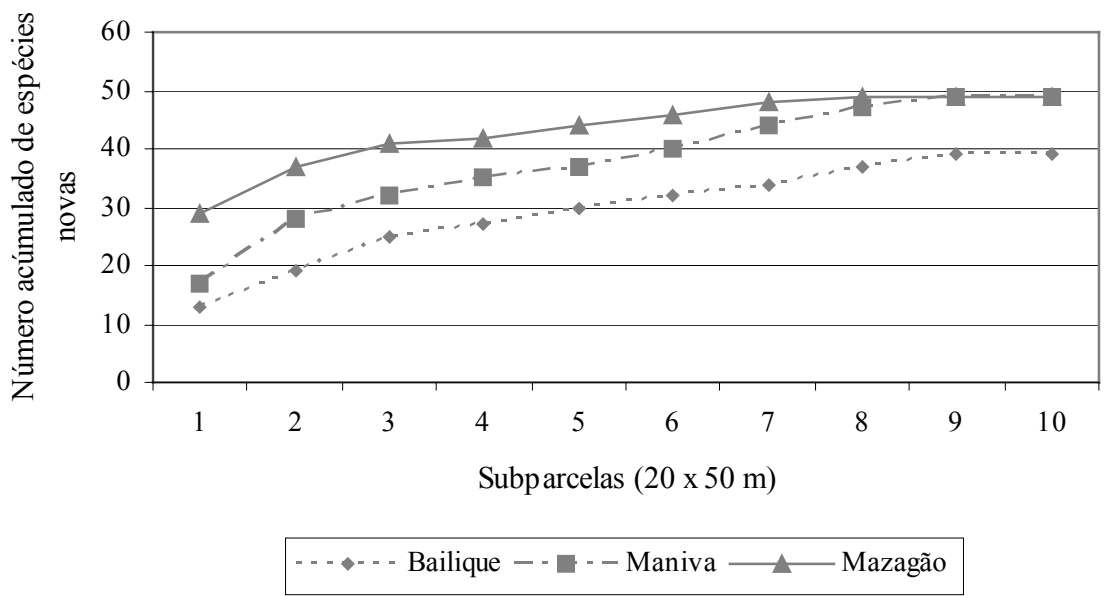

Figura 2. Curva espécie/área na várzea alta.

Figure 2. Species/area curve in high floodplain. 
Tabela 1. Famílias e espécies com nomes científicos e comuns, ocorrência e índices de Mc Guinnes nas áreas estudadas.

Table 1. Tree families and species with their scientific and common names, occurrence and Mc Guinnes index in the studied areas.

\begin{tabular}{|c|c|c|c|c|c|c|c|c|}
\hline \multirow{3}{*}{ FAMÍLIAS } & \multirow{3}{*}{ ESPÉCIES } & \multirow{3}{*}{ NOME POPULAR } & \multicolumn{6}{|c|}{ ÍNDICE DE MC GUINNES } \\
\hline & & & \multicolumn{2}{|c|}{ Furo do Mazagão } & \multicolumn{2}{|r|}{ Rio Maniva } & \multicolumn{2}{|r|}{ Bailique } \\
\hline & & & IGA & Distribuição & IGA & Distribuição & IGA & Distribuição \\
\hline$\overline{\text { Annonaceae }}$ & Guateria poeppigiana Mart. & Envira Preta & 1,17 & Agrupamento & 0,84 & Uniforme & $*$ & \\
\hline Anacardiaceae & Spondias mombin $\mathrm{L}$. & Taperebá & 0,95 & Uniforme & $*$ & & 1,90 & Agrupamento \\
\hline Bombacaceae & Matisia paraensis Huber. & Cupuçurana & 0,81 & Uniforme & 0,85 & Uniforme & 0,90 & Uniforme \\
\hline$"$ & Quararibea guianensis Aubl. & Inajarana & 0,87 & Uniforme & 0,69 & Uniforme & $*$ & \\
\hline Burceraceae & Protium spruceanum Engl. & Breu branco & 0,95 & Uniforme & $*$ & & $*$ & \\
\hline Caesalpiniaceae & Campsiandra laurifolia Benth. & Acapurana & 0,84 & Uniforme & * & & 0,78 & Uniforme \\
\hline " & Hymenaea oblongifolia Huber & Jutaí da folha fina & 0,95 & Uniforme & 0,95 & Uniforme & $*$ & \\
\hline$"$ & Macrolobium acaciaefolium Benth. & Arapari & 0,98 & Uniforme & $*$ & & * & \\
\hline$"$ & Macrolobium augustifolium R.S.Cowan & Jutaí da folha larga & $*$ & & 0,90 & Uniforme & * & \\
\hline$"$ & Mora paraensis Ducke & Pracuúba & 6,08 & Contagiosa & 1,89 & Agrupamento & 0,39 & Uniforme \\
\hline$"$ & Swartzia cardiosperma Spr. Ex. Benth. & Pacapeuá & 1,01 & Agrupamento & 2,15 & Contagiosa & 1,68 & Agrupamento \\
\hline Caryocaceae & Caryocar glabrum (Aubl.) Pers. & Piquiarana & 0,90 & Uniforme & 0,84 & Uniforme & $*$ & \\
\hline Chrysobalanaceae & Licania heteromorpha Benth. & Macucu & 9,41 & Contagiosa & 0,72 & Uniforme & $*$ & \\
\hline$"$ & Licania kunthiana & Cariperana & $*$ & & 0,95 & Uniforme & * & \\
\hline$"$ & Licania macrophylla Benth. & Anoerá & 3,79 & Contagiosa & 1,62 & Agrupamento & 0,95 & Uniforme \\
\hline$"$ & Parinari excelsa Sabine & Paranari & $*$ & & $*$ & & 0,95 & Uniforme \\
\hline Clusiaceae & Calophyllum brasiliensis Cambess. & Jacareúba & 0,84 & Uniforme & 0,95 & Uniforme & $*$ & \\
\hline$"$ & Caraipa grandiflora Mart. & Tamaquaré & 0,78 & Uniforme & 1,64 & Agrupamento & 0,87 & Uniforme \\
\hline$"$ & Rheedia acuminata Planchon et Triana. & Bacurizinho & $*$ & & 0,90 & Uniforme & $*$ & \\
\hline$"$ & Rheedia macrophylla (Mart.) Pl. et Jr. & Bacuri pari & $*$ & & 0,90 & Uniforme & 1,12 & Agrupamento \\
\hline$"$ & Symphonia globulifera L.F. & Anani & 1,12 & Agrupamento & 0,52 & Uniforme & 1,00 & Aleatória \\
\hline Combretaceae & Combretum cacoucia Excell \& Sandw & Ioióca & $*$ & & 0,72 & Uniforme & 1,01 & Agrupamento \\
\hline " & Terminalia dichotoma $\mathrm{G}$. Meyer & Cuiarana & 1,90 & Agrupamento & $*$ & & $*$ & \\
\hline Euphorbiaceae & Hevea brasiliensis Mull. Arg. & Seringueira & 0,95 & Uniforme & 1,01 & Agrupamento & 0,98 & Uniforme \\
\hline Fabaceae & Dipteryx sp & Cumarurana & 0,66 & Uniforme & 0,84 & Uniforme & 0,98 & Uniforme \\
\hline$"$ & Platymiscium filipes Benth. & Macacaúba & 0,95 & Uniforme & $*$ & & 1,57 & Agrupamento \\
\hline$"$ & Pterocarpus amazonicus Huber. & Mututi & 1,41 & Agrupamento & 0,81 & Uniforme & 0,84 & Uniforme \\
\hline
\end{tabular}


continuação

\begin{tabular}{|c|c|c|c|c|c|c|c|c|}
\hline \multirow{3}{*}{ FAMÍLIAS } & \multirow{3}{*}{ ESPÉCIES } & \multirow{3}{*}{ NOME POPULAR } & \multicolumn{6}{|c|}{ ÍNDICE DE MC GUINNES } \\
\hline & & & \multicolumn{2}{|c|}{ Furo do Mazagão } & \multicolumn{2}{|r|}{ Rio Maniva } & \multicolumn{2}{|r|}{ Bailique } \\
\hline & & & IGA & Distribuição & IGA & Distribuição & IGA & Distribuição \\
\hline \multirow{2}{*}{$\begin{array}{l}\text { Fabaceae } \\
"\end{array}$} & Pterocarpus officinalis Jacq & Mututirana & 0,98 & Uniforme & 1,68 & Agrupamento & 0,95 & Uniforme \\
\hline & Vatairea guianensis Aubl. & Faveira & 0,90 & Uniforme & 1,79 & Agrupamento & $*$ & \\
\hline Hernandiaceae & Hernandia guianensis Aubl. & Ventosa & $*$ & & 0,87 & Uniforme & * & \\
\hline Humiriaceae & Saccoglottis guianensis Aubl. & Uxirana & 0,95 & Uniforme & $*$ & & $*$ & \\
\hline Icacinaceae & Dendrobangia boliviana Rusby & Caferana & $*$ & & 0,95 & Uniforme & $*$ & \\
\hline \multirow{2}{*}{$\begin{array}{l}\text { Lauraceae } \\
"\end{array}$} & Licaria canella (Meiss.) Kosterm & Louro pretinho & $*$ & & 0,95 & Uniforme & $*$ & \\
\hline & Licaria mahuba Kosterm & Maúba & 1,17 & Agrupamento & 0,76 & Uniforme & $*$ & \\
\hline \multirow{2}{*}{$\begin{array}{l}\text { Lecythidaceae } \\
"\end{array}$} & Couroupita guianensis Aubl. & Castanha de macaco & $*$ & & $*$ & & 0,95 & Uniforme \\
\hline & Gustavia augusta L. & Jenipaparana & 1,79 & Agrupamento & $*$ & & $*$ & \\
\hline \multirow{2}{*}{$\begin{array}{l}\text { Melastomataceae } \\
"\end{array}$} & Miconia ceramicarpa Cogn. & papa-terra & 1,55 & Agrupamento & $*$ & & $*$ & \\
\hline & Mouriri acutiflora Naud. & Camutim & 0,95 & Uniforme & $*$ & & 1,12 & Agrupamento \\
\hline \multirow{2}{*}{$\begin{array}{l}\text { Meliaceae } \\
"\end{array}$} & Carapa guianensis Aubl. & Andiroba & 1,17 & Agrupamento & 0,54 & Uniforme & 0,75 & Uniforme \\
\hline & Trichilia surinamensis (Miq.) C.DC. & Marajoão & $*$ & & $*$ & & 0,75 & Uniforme \\
\hline \multirow{2}{*}{$\begin{array}{l}\text { Mimosaceae } \\
"\end{array}$} & Inga $\mathrm{sp} 1$ & Ingá branco & 0,72 & Uniforme & 1,34 & Agrupamento & $*$ & \\
\hline & Inga $\mathrm{sp} 2$ & Ingá & 1,40 & Agrupamento & 0,95 & Uniforme & 0,95 & Uniforme \\
\hline \multirow{2}{*}{ " } & Pentaclethra macroloba (Willd) O. Kuntze & Pracaxi & 1,30 & Agrupamento & 1,61 & Agrupamento & 1,43 & Agrupamento \\
\hline & Pithecellobium inaequale (H.B.K.) Benth. & Jaranduba da mata & 0,46 & Uniforme & 1,17 & Agrupamento & 0,90 & Uniforme \\
\hline \multirow{2}{*}{ " } & Pithecellobium sp & Jaranduba & 0,90 & Uniforme & $*$ & & 1,90 & Agrupamento \\
\hline & Swartzia acuminata Willd & Pitaíca & 0,95 & Uniforme & 0,43 & Uniforme & $*$ & \\
\hline \multirow{5}{*}{$\begin{array}{l}\text { Moraceae } \\
\text { Myristicaceae } \\
\text { Myrsinaceae } \\
\text { Myrtaceae } \\
\text { " }\end{array}$} & Olmedia caloneura Huber. & Muiratinga & 0,98 & Uniforme & 0,90 & Uniforme & $*$ & \\
\hline & Virola surinamensis (Rol.) Warb. & Virola & 1,25 & Agrupamento & 0,81 & Uniforme & 0,58 & Uniforme \\
\hline & Spdesc & Olho de galega & $*$ & & 0,84 & Uniforme & $*$ & \\
\hline & Calyptranthes speciosa Sagot. & Goiabarana & 0,90 & Uniforme & 0,95 & Uniforme & 1,12 & Agrupamento \\
\hline & Eugenia browsnbergii Amshoff & Goiaba braba & 1,68 & Agrupamento & 1,17 & Agrupamento & 1,50 & Agrupamento \\
\hline \multirow{2}{*}{$\begin{array}{l}\text { Rubiaceae } \\
\text { " }\end{array}$} & Alibertia edulis A. Rich. & Açaí pretinho & 0,95 & Uniforme & 0,95 & Uniforme & $*$ & \\
\hline & Callycophyllum spruceanum Benth. & Pau mulato & 0,95 & Uniforme & 0,90 & Uniforme & $*$ & \\
\hline Rutaceae & Metrodorea flavida Krause. & Laranjinha & $*$ & & 0,95 & Uniforme & 0,29 & Uniforme \\
\hline
\end{tabular}


continuação ...

\begin{tabular}{|c|c|c|c|c|c|c|c|c|}
\hline \multirow{3}{*}{ FAMÍLIAS } & \multirow{3}{*}{ ESPÉCIES } & \multirow{3}{*}{ NOME POPULAR } & \multicolumn{6}{|c|}{ ÍNDICE DE MC GUINNES } \\
\hline & & & \multicolumn{2}{|c|}{ Furo do Mazagão } & \multicolumn{2}{|r|}{ Rio Maniva } & \multicolumn{2}{|r|}{ Bailique } \\
\hline & & & IGA & Distribuição & IGA & Distribuição & IGA & Distribuição \\
\hline Sapotaceae & Crysophyllum excelsum Huber. & Guajaraí & $*$ & & $*$ & & 0,75 & Uniforme \\
\hline & Pouteria bilocularis (Winkler) Baehni & Abiurana & 1,40 & Agrupamento & $*$ & & $*$ & \\
\hline$"$ & Pouteria sagotiana (Baill) Eyma. & Maçaranduba & 0,95 & Uniforme & 0,90 & Uniforme & $*$ & \\
\hline Sterculiaceae & Herrania mariae (Mart.) Dene. & Cacau jacaré & $*$ & & $*$ & & 0,84 & Uniforme \\
\hline & Sterculia speciosa K. Schum & Capoteiro & 1,90 & Agrupamento & 0,87 & Uniforme & 0,72 & Uniforme \\
\hline$"$ & Theobroma cacao L. & Cacau & 0,95 & Uniforme & $*$ & & $*$ & \\
\hline Tiliaceae & Apeiba burchelii Sprague. & Chapéu de sol & $*$ & & 0,95 & Uniforme & $*$ & \\
\hline Arecaceae & Astrocaryum murumuru Mart. & Murumuru & 3,13 & Contagiosa & 3,11 & Contagiosa & 4,21 & Contagiosa \\
\hline$"$ & Attalea excelsa Mart. & Urucuri & $*$ & & 0,90 & Uniforme & 1,11 & Agrupamento \\
\hline$"$ & Bactris maraja Mart. & Marajá & 0,95 & Uniforme & $*$ & & $*$ & \\
\hline$"$ & Manicaria saccifera Gaertn. & Buçu & 1,37 & Agrupamento & 2,21 & Contagiosa & 1,18 & Agrupamento \\
\hline$"$ & Mauritia flexuosa L. & Buriti & $*$ & & 0,72 & Uniforme & 0,72 & Uniforme \\
\hline$"$ & Oenocarpus distichus Mart. & Bacaba & * & & $*$ & & 0,95 & Uniforme \\
\hline$"$ & Socratea exhorriza (Mart.) Wendl. & Paxiúba & $*$ & & 3,80 & Contagiosa & $*$ & \\
\hline$"$ & Euterpe oleracea Mart. & Açaí & 4,69 & Contagiosa & 3,65 & Contagiosa & 5,15 & Contagiosa \\
\hline
\end{tabular}

Uniforme $=$ Espécies com distribuição uniforme, Aleatória $=$ Espécies com tendência de distribuição aleatória,

Agrupamento $=$ Espécies com tendência ao agrupamento, Contagiosa $=$ Espécies com distribuição contagiosa.

Uniform $=$ species with uniform distribution, Random $=$ Species with random distribution tendency

Groupment $=$ Species with groupment tendency, Contagious $=$ Species with contagious distribution .

* Espécie ausente 
Observa-se, ainda, nos três locais, uma tendência inversa quando se considera a população de açaizeiros e das demais espécies, pois, em vários momentos, o aumento de uma implica na diminuição das outras (Figura 3), fato este também observado por Dubois et al. (1996) e Queiroz e Mochiutti (2000). Esta relação é alterada nas ocasiões em que ocorre exploração de madeira, limpezas nos açaizais ou corte excessivo de palmito. $\mathrm{Na}$ ausência de intervenção humana, a relação se altera com a regeneração natural, cuja sucessão favorece as espécies dicotiledôneas, que se tornam mais numerosas até atingir a fase de clímax da floresta.

Tanto no furo do Mazagão quanto no rio Maniva e em Bailique, Arecaceae foi a família que apresentou maior densidade absoluta, bem como suas duas espécies principais, E. oleraceae (216, 168 e $237 \mathrm{ind} / \mathrm{ha})$ e $A$. murumuru (144, 143 e $194 \mathrm{ind} /$ ha). Com relação às dicotiledôneas, Caesalpiniaceae foi a melhor representada, com a espécie $M$. paraensis $(140,87$ e $18 \mathrm{ind} / \mathrm{ha})$, que foi também $1^{\mathrm{a}}, 2^{\mathrm{a}}$ e $3^{\mathrm{a}}$ em dominância relativa, com 22,08\%; 11,60\% e 8,73\% (Tabela 2).

As densidades de touceiras de açaizeiros representaram 24,05\%, 19,53\% e 32,87\% da população total de espécies arbóreas, respectivamente, para Mazagão, Maniva e Bailique. Estes resultados são compatíveis com os encontrados por Anderson et al. (1985) para as áreas de várzea, mas diferem dos resultados mencionados por Nogueira (1999), para as áreas de açaizais manejadas no município de Igarapé Miri, no Estado do Pará, e dos resultados encontrados por Rabelo (1999), para as áreas de Mazagão e Lontra da Pedreira no estado do Amapá.

Em estudo de estrutura e composição florística realizado por Rabelo (1999), em duas regiões no estado do Amapá, no qual foram inventariadas cinco parcelas de um hectare em cada uma, sendo medidas todas as árvores com DAP $\geq 5,0 \mathrm{~cm}$, as espécies E. oleracea, Astrocaryum murumuru, Licania heteromorpha e Calycophyllum spruceanum apresentaram maior valor de importância nas duas áreas amostradas, com valores aproximadamente semelhantes. Ao todo, no estudo de Rabelo, foram encontradas 102 espécies distribuídas em 34 famílias, além de 12 espécies e três famílias não identificadas.

De acordo com Ducke e Black (1954), a Amazônia brasileira é um dos dois centros mundiais de distribuição das palmeiras, porém não uniforme; cabe à parte ocidental o maior número de espécies, sendo o estuário amazônico mais rico em indivíduos. Ainda, segundo os autores, depois das palmeiras, o elemento mais importante na fisionomia da flora hileana é constituído por leguminosas. Os resultados encontrados no presente estudo são compatíveis com esses resultados: do total de indivíduos arbóreos, as palmeiras constituíram 41\%, 43\% e 70\%, respectivamente, para Mazagão, Maniva e Bailique, enquanto as leguminosas constituíram $31 \%, 28 \%$ e $12 \%$ do total de indivíduos.

Ainda hoje é comum adotar-se o "nomina conservada" para leguminosae. Mesmo assim, observa-se, praticamente, o mesmo resultado: as palmeiras se destacam pela densidade absoluta nos três locais e só perdem para as leguminosas em dominância e em valor de importância no Mazagão. No rio Maniva, praticamente não houve diferença. No Bailique a superioridade das palmeiras é grande, com 507, $54,7 \%$ e 150,1 , respectivamente, para densidade absoluta, dominância relativa e valor de importância, enquanto as leguminosas apresentam $83,24,5 \%$ e 59,8 (Tabela 3). Com relação ao número de espécies, as palmeiras apresentam 4, 7 e 7, respectivamente no furo do Mazagão, rio Maniva e Bailique e as leguminosas 15, 13 e 10, o que mostra a superioridade das leguminosas. Esses resultados foram encontrados também por Ducke e Black (1954), Bentes-Gama (2000) e Jardim e Vieira (2001) na várzea alta. 

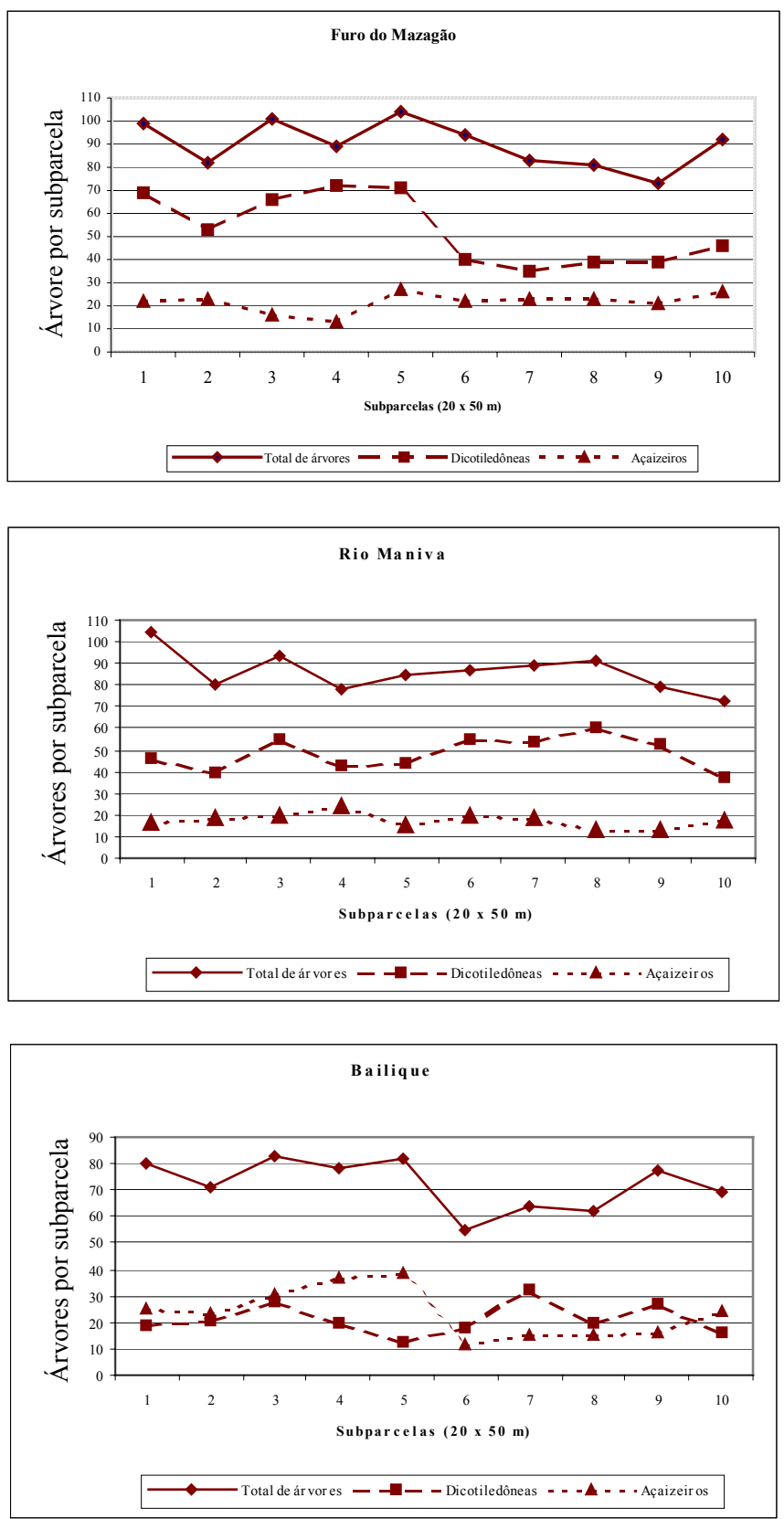

Figura 3. Competição entre Euterpe oleracea Mart. e Dicotiledôneas nas áreas de estudo.

Figure 3. Competition among Euterpe oleracea Mart. and Dicotyledonous at the study areas.

Na Amazônia, Melastomataceae e Myrtaceae são muito menos importante que no sul e leste tropical e subtropical do Brasil, apesar da sua grande freqüência em formações secundárias (Ducke e Black, 1954). Estas observações são confirmadas com os resultados encontrados no presente trabalho (Tabela 3), no qual foram encontradas duas espécies para a família Melastomataceae, com destaque para Miconia ceramicarpa (papa-terra), árvore do estrato inferior, que tem sua presença ligada à ação antrópica. O mesmo pode ser atribuído à espécie Eugenia browsbergii (goiabinha) da família Myrtaceae. 
Tabela 2. Freqüência, densidade, dominância, valor de importância e densidade absoluta total por espécie nas três áreas.

Table 2. Frequence, density, dominance, importance value and total dominance absolute for tree species in the three areas.

\begin{tabular}{|c|c|c|c|c|c|c|c|c|c|c|c|c|c|c|c|c|c|c|c|c|c|c|}
\hline \multirow[b]{2}{*}{ DAT } & \multirow[b]{2}{*}{ ESPÉCIES } & \multicolumn{7}{|c|}{ Furo do Mazagão } & \multicolumn{7}{|c|}{ Rio Maniva } & \multicolumn{7}{|c|}{ Bailique } \\
\hline & & FA & FR & DA & $\overline{\mathrm{DR}}$ & DoA & DoR & $\mathrm{VI}$ & FA & FR & $\mathrm{DA}$ & $\overline{\mathrm{DR}}$ & DoA & DoR & $\mathrm{VI}$ & FA & FR & $\mathrm{DA}$ & $\overline{\mathrm{DR}}$ & DoA & DoR & $\mathrm{VI}$ \\
\hline 621 & Euterpe oleraceae & 100 & 5,08 & 216 & 24,08 & 5,74 & 16,78 & 45,94 & 100 & 4,42 & 168 & 19,53 & 5,94 & 16,24 & 40,20 & 100 & 5,68 & 237 & 32,87 & 8,92 & 29,66 & 68,22 \\
\hline 481 & Astrocarium murun & 100 & 5,08 & 144 & 16,05 & 2,43 & 7,11 & 28,24 & 100 & 4,42 & 143 & 16,63 & 2,31 & 6,32 & 27,37 & 100 & 5,68 & 194 & 26,91 & 2,14 & 7,12 & 39,71 \\
\hline 245 & Mora paraensis & 90 & 4,57 & 140 & 15,61 & 7,50 & 21,94 & 42,12 & 100 & 4,42 & 87 & 10,12 & 4,19 & 11,46 & 26,01 & 100 & 5,68 & 18 & 2,50 & 2,69 & 8,94 & 17,12 \\
\hline 167 & Pentaclethra macroloba & 100 & 5,08 & 60 & 6,69 & 1,56 & 4,57 & 16,33 & 100 & 4,42 & 74 & 8,60 & 2,13 & 5,82 & 18,85 & 90 & 5,11 & 33 & 4,58 & 1,78 & 5,94 & 15,63 \\
\hline 88 & Licania macrophylla & 80 & 4,06 & 61 & 6,80 & 1,89 & 5,54 & 16,40 & 80 & 3,54 & 26 & 3,02 & 1,49 & 4,08 & 10,65 & 10 & 0,57 & 1 & 0,14 & 0,02 & 0,08 & 0,78 \\
\hline 77 & Manicaria saccifera & 40 & 2,03 & 7 & 0,78 & 0,37 & 1,07 & 3,88 & 90 & 3,98 & 51 & 5,93 & 2,25 & 6,15 & 16,07 & 80 & 4,55 & 19 & 2,64 & 0,84 & 2,78 & 9,96 \\
\hline 61 & Carapa guianensis & 90 & 4,57 & 27 & 3,01 & 1,42 & 4,16 & 11,74 & 100 & 4,42 & 25 & 2,91 & 1,41 & 3,85 & 11,19 & 70 & 3,98 & 9 & 1,25 & 0,28 & 0,94 & 6,17 \\
\hline 54 & Matisia paraensis & 80 & 4,06 & 13 & 1,45 & 0,57 & 1,68 & 7,19 & 100 & 4,42 & 39 & 4,53 & 2,27 & 6,20 & 15,16 & 20 & 1,14 & 2 & 0,28 & 0,01 & 0,04 & 1,45 \\
\hline 51 & Symphonia globulifera & 30 & 1,52 & 4 & 0,45 & 0,32 & 0,94 & 2,90 & 100 & 4,42 & 24 & 2,79 & 1,53 & 4,17 & 11,39 & 90 & 5,11 & 23 & 3,19 & 1,83 & 6,10 & 14,40 \\
\hline 50 & Pithecellobium inaequale & 100 & 5,08 & 21 & 2,34 & 0,14 & 0,42 & 7,83 & 90 & 3,98 & 27 & 3,14 & 0,26 & 0,72 & 7,85 & 20 & 1,14 & 2 & 0,28 & 0,01 & 0,02 & 1,44 \\
\hline 35 & Virola surinamensis & 70 & 3,55 & 15 & 1,67 & 0,78 & 2,29 & 7,51 & 80 & 3,54 & 13 & 1,51 & 0,99 & 2,71 & 7,76 & 70 & 3,98 & 7 & 0,97 & 0,30 & 0,99 & 5,94 \\
\hline 33 & Pterocarpus amazonicu & 70 & 3,55 & 17 & 1,90 & 0,90 & 2,64 & 8,09 & 80 & 3,54 & 13 & 1,51 & 0,32 & 0,87 & 5,92 & 30 & 1,70 & 3 & 0,42 & 0,33 & 1,09 & 3,21 \\
\hline 30 & Eugenia browsnbergii & 30 & 1,52 & 6 & 0,67 & 0,03 & 0,07 & 2,27 & 40 & 1,77 & 6 & 0,70 & 0,06 & 0,17 & 2,64 & 70 & 3,98 & 18 & 2,50 & 0,13 & 0,45 & 6,92 \\
\hline 27 & Caraipa grandiflora & 40 & 2,03 & 4 & 0,45 & 0,06 & 0,17 & 2,64 & 60 & 2,65 & 15 & 1,74 & 0,22 & 0,61 & 5,01 & 60 & 3,41 & 8 & 1,11 & 0,20 & 0,67 & 5,19 \\
\hline 24 & Swartzia cardiosperma & 50 & 2,54 & 7 & 0,78 & 0,33 & 0,97 & 4,29 & 40 & 1,77 & 11 & 1,28 & 0,80 & 2,18 & 5,23 & 30 & 1,70 & 6 & 0,83 & 0,05 & 0,16 & 2,69 \\
\hline 17 & Hevea brasiliensis & 10 & 0,51 & 1 & 0,11 & 0,00 & 0,01 & 0,63 & 50 & 2,21 & 7 & 0,81 & 0,63 & 1,71 & 4,74 & 60 & 3,41 & 9 & 1,25 & 0,82 & 2,71 & 7,37 \\
\hline 16 & Dipteryx $s p$ & 70 & 3,55 & 8 & 0,89 & 0,08 & 0,23 & 4,67 & 30 & 1,33 & 3 & 0,35 & 0,24 & 0,66 & 2,34 & 40 & 2,27 & 5 & 0,69 & 0,42 & 1,41 & 4,38 \\
\hline 16 & Pterocarpus officinalis & 60 & 3,05 & 9 & 1,00 & 0,47 & 1,38 & 5,43 & 30 & 1,33 & 6 & 0,70 & 0,25 & 0,67 & 2,70 & 10 & 0,57 & 1 & 0,14 & 0,09 & 0,31 & 1,02 \\
\hline 13 & Sterculia speciosa & 10 & 0,51 & 2 & 0,22 & 0,04 & 0,12 & 0,85 & 50 & 2,21 & 6 & 0,70 & 0,15 & 0,40 & 3,31 & 50 & 2,84 & 5 & 0,69 & 0,35 & 1,15 & 4,68 \\
\hline 7 & Calyptranthes speciosa & 20 & 1,02 & 2 & 0,22 & 0,01 & 0,02 & 1,26 & 10 & 0,44 & 1 & 0,12 & 0,01 & 0,02 & 0,58 & 30 & 1,70 & 4 & 0,55 & 0,07 & 0,22 & 2,48 \\
\hline 7 & Inga sp2 & 30 & 1,52 & 5 & 0,56 & 0,21 & 0,61 & 2,69 & 10 & 0,44 & 1 & 0,12 & 0,01 & 0,02 & 0,58 & 10 & 0,57 & 1 & 0,14 & 0,18 & 0,60 & 1,30 \\
\hline
\end{tabular}

continua 
continuação ..

\begin{tabular}{|c|c|c|c|c|c|c|c|c|c|c|c|c|c|c|c|c|c|c|c|c|c|c|}
\hline \multirow[b]{2}{*}{ DAT } & \multirow[b]{2}{*}{ ESPÉCIES } & \multicolumn{7}{|c|}{ Furo do Mazagão } & \multicolumn{7}{|c|}{ Rio Maniva } & \multicolumn{7}{|c|}{ Bailique } \\
\hline & & FA & FR & $\mathrm{DA}$ & DR & DoA & DoR & VI & FA & FR & $\mathrm{DA}$ & DR & DoA & DoR & VI & FA & FR & $\mathrm{DA}$ & DR & DoA & DoR & VI \\
\hline 52 & Quararibea guianensis & 90 & 4,57 & 20 & 2,23 & 0,49 & 1,44 & 8,24 & 100 & 4,42 & 32 & 3,72 & 0,59 & 1,61 & 9,76 & & & & & & & \\
\hline 26 & Licania heteromorpha & 20 & 1,02 & 21 & 2,34 & 2,11 & 6,16 & 9,52 & 50 & 2,21 & 5 & 0,58 & 1,10 & 3,02 & 5,81 & & & & & & & \\
\hline 13 & Licaria mahuba & 40 & 2,03 & 6 & 0,67 & 0,41 & 1,19 & 3,89 & 60 & 2,65 & 7 & 0,81 & 0,39 & 1,06 & 4,53 & & & & & & & \\
\hline 11 & Swartzia acuminata & 10 & 0,51 & 1 & 0,11 & 0,00 & 0,00 & 0,62 & 90 & 3,98 & 10 & 1,16 & 2,52 & 6,88 & 12,03 & & & & & & & \\
\hline 9 & Guateria poeppigiana & 40 & 2,03 & 6 & 0,67 & 0,10 & 0,29 & 2,99 & 30 & 1,33 & 3 & 0,35 & 0,03 & 0,10 & 1,77 & & & & & & & \\
\hline 8 & Inga spl & 50 & 2,54 & 5 & 0,56 & 0,02 & 0,05 & 3,14 & 20 & 0,88 & 3 & 0,35 & 0,04 & 0,10 & 1,33 & & & & & & & \\
\hline 7 & Olmedia caloneura & 40 & 2,03 & 5 & 0,56 & 0,88 & 2,58 & 5,17 & 20 & 0,88 & 2 & 0,23 & 0,41 & 1,13 & 2,25 & & & & & & & \\
\hline 6 & Vatairea guianensis & 20 & 1,02 & 2 & 0,22 & 0,50 & 1,45 & 2,69 & 20 & 0,88 & 4 & 0,47 & 0,60 & 1,65 & 3,00 & & & & & & & \\
\hline 5 & Caryocar glabrum & 20 & 1,02 & 2 & 0,22 & 0,03 & 0,08 & 1,31 & 30 & 1,33 & 3 & 0,35 & 0,23 & 0,64 & 2,32 & & & & & & & \\
\hline 4 & Calophyllum brasiliensis & 30 & 1,52 & 3 & 0,33 & 0,15 & 0,43 & 2,29 & 10 & 0,44 & 1 & 0,12 & 0,17 & 0,45 & 1,01 & & & & & & & \\
\hline 3 & Callycophyllum spruceanum & 10 & 0,51 & 1 & 0,11 & 0,23 & 0,67 & 1,29 & 20 & 0,88 & 2 & 0,23 & 0,80 & 2,19 & 3,31 & & & & & & & \\
\hline 3 & Pouteria sagotiana & 10 & 0,51 & 1 & 0,11 & 0,01 & 0,04 & 0,66 & 20 & 0,88 & 2 & 0,23 & 0,84 & 2,30 & 3,42 & & & & & & & \\
\hline 2 & Alibertia edulis & 10 & 0,51 & 1 & 0,11 & 0,00 & 0,01 & 0,63 & 10 & 0,44 & 1 & 0,12 & 0,00 & 0,01 & 0,56 & & & & & & & \\
\hline 2 & Hymenaea oblongifolia & 10 & 0,51 & 1 & 0,11 & 0,02 & 0,07 & 0,69 & 10 & 0,44 & 1 & 0,12 & 0,01 & 0,01 & 0,57 & & & & & & & \\
\hline 9 & Platymiscium filipes & 10 & 0,51 & 1 & 0,11 & 1,12 & 3,28 & 3,90 & & & & & & & & 40 & 2,27 & 8 & 1,11 & 0,84 & 2,80 & 6,18 \\
\hline 7 & Campsiandra laurifolia & 30 & 1,52 & 3 & 0,33 & 0,29 & 0,86 & 2,71 & & & & & & & & 40 & 2,27 & 4 & 0,55 & 0,80 & 2,65 & 5,47 \\
\hline 5 & Mouriri acutiflora & 10 & 0,51 & 1 & 0,11 & 0,05 & 0,14 & 0,76 & & & & & & & & 30 & 1,70 & 4 & 0,55 & 0,05 & 0,15 & 2,41 \\
\hline 4 & Pithecellobium sp & 20 & 1,02 & 2 & 0,22 & 0,01 & 0,03 & 1,27 & & & & & & & & 10 & 0,57 & 2 & 0,28 & 0,16 & 0,54 & 1,39 \\
\hline 3 & Spondias Mombim & 10 & 0,51 & 1 & 0,11 & 0,07 & 0,21 & 0,83 & & & & & & & & 10 & 0,57 & 2 & 0,28 & 1,07 & 3,57 & 4,41 \\
\hline 53 & Attalea excelsa & & & & & & & & 20 & 0,88 & 2 & 0,23 & 0,19 & 0,51 & 1,63 & 100 & 5,68 & 51 & 7,07 & 4,04 & 13,45 & 26,20 \\
\hline 12 & Combretum cacoucia & & & & & & & & 50 & 2,21 & 5 & 0,58 & 0,02 & 0,06 & 2,86 & 50 & 2,84 & 7 & 0,97 & 0,02 & 0,07 & 3,88 \\
\hline 10 & Mauritia flexuosa & & & & & & & & 50 & 2,21 & 5 & 0,58 & 0,46 & 1,25 & 4,05 & 50 & 2,84 & 5 & 0,69 & 0,50 & 1,65 & 5,19 \\
\hline 6 & Rheedia macrophylla & & & & & & & & 20 & 0,88 & 2 & 0,23 & 0,02 & 0,06 & 1,17 & 30 & 1,70 & 4 & 0,55 & 0,06 & 0,20 & 2,46 \\
\hline 3 & Metrodorea flavida & & & & & & & & 10 & 0,44 & 1 & 0,12 & 0,00 & 0,01 & 0,57 & 50 & 2,84 & 2 & 0,28 & 0,01 & 0,02 & 3,14 \\
\hline 25 & Miconia ceramicarpa & 80 & 4,06 & 25 & 2,79 & 0,27 & 0,79 & 7,64 & & & & & & & & & & & & & & \\
\hline
\end{tabular}

continua ... 
continuação ...

\begin{tabular}{|c|c|c|c|c|c|c|c|c|c|c|c|c|c|c|c|c|c|c|c|c|c|c|}
\hline \multirow[b]{2}{*}{ DAT } & \multirow[b]{2}{*}{ ESPÉCIES } & \multicolumn{7}{|c|}{ Furo do Mazagão } & \multicolumn{7}{|c|}{ Rio Maniva } & \multicolumn{7}{|c|}{ Bailique } \\
\hline & & FA & FR & DA & DR & DoA & DoR & VI & FA & FR & DA & $\mathrm{DR}$ & DoA & DoR & VI & FA & FR & $\mathrm{DA}$ & $\mathrm{DR}$ & DoA & DoR & VI \\
\hline 5 & Macrolobium acaciaefolium & 40 & 2,03 & 5 & 0,56 & 2,18 & 6,39 & 8,97 & & & & & & & & & & & & & & \\
\hline 5 & Pouteria bilocularis & 30 & 1,52 & 5 & 0,56 & 0,27 & 0,80 & 2,88 & & & & & & & & & & & & & & \\
\hline 4 & Gustavia augusta & 20 & 1,02 & 4 & 0,45 & 0,08 & 0,24 & 1,70 & & & & & & & & & & & & & & \\
\hline 2 & Terminalia dichotoma & 10 & 0,51 & 2 & 0,22 & 0,01 & 0,02 & 0,76 & & & & & & & & & & & & & & \\
\hline 1 & Bactris maraja & 10 & 0,51 & 1 & 0,11 & 0,00 & 0,00 & 0,62 & & & & & & & & & & & & & & \\
\hline 1 & Protium spruceanum & 10 & 0,51 & 1 & 0,11 & 0,00 & 0,01 & 0,63 & & & & & & & & & & & & & & \\
\hline 1 & Saccoglottis guianensis & 10 & 0,51 & 1 & 0,11 & 0,01 & 0,04 & 0,65 & & & & & & & & & & & & & & \\
\hline 1 & Theobroma cacao & 10 & 0,51 & 1 & 0,11 & 0,01 & 0,02 & 0,63 & & & & & & & & & & & & & & \\
\hline 8 & Hernandia guianensis & & & & & & & & 60 & 2,65 & 8 & 0,93 & 0,17 & 0,46 & 4,05 & & & & & & & \\
\hline 4 & Socratea exhorriza & & & & & & & & 10 & 0,44 & 4 & 0,47 & 0,06 & 0,17 & 1,08 & & & & & & & \\
\hline 3 & Spdesc & & & & & & & & 30 & 1,33 & 3 & 0,35 & 0,01 & 0,02 & 1,70 & & & & & & & \\
\hline 2 & Macrolobium augustifolium & & & & & & & & 20 & 0,88 & 2 & 0,23 & 0,02 & 0,06 & 1,17 & & & & & & & \\
\hline 2 & Rheedia acuminata & & & & & & & & 20 & 0,88 & 2 & 0,23 & 0,01 & 0,02 & 1,13 & & & & & & & \\
\hline 1 & Apeiba burchelii & & & & & & & & 10 & 0,44 & 1 & 0,12 & 0,28 & 0,78 & 1,34 & & & & & & & \\
\hline 1 & Dendrobangia boliviana & & & & & & & & 10 & 0,44 & 1 & 0,12 & 0,01 & 0,03 & 0,59 & & & & & & & \\
\hline 1 & Licania kunthiana & & & & & & & & 10 & 0,44 & 1 & 0,12 & 0,05 & 0,13 & 0,69 & & & & & & & \\
\hline 1 & Licaria canella & & & & & & & & 10 & 0,44 & 1 & 0,12 & 0,08 & 0,23 & 0,79 & & & & & & & \\
\hline 12 & Trichilia surinamensis & & & & & & & & & & & & & & & 80 & 4,55 & 12 & 1,66 & 0,25 & 0,83 & 7,04 \\
\hline 9 & Crysophyllum excelsum & & & & & & & & & & & & & & & 70 & 3,98 & 9 & 1,25 & 0,41 & 1,37 & 6,60 \\
\hline 3 & Herrania mariae & & & & & & & & & & & & & & & 30 & 1,70 & 3 & 0,42 & 0,01 & 0,02 & 2,14 \\
\hline 1 & Couroupita guianensis & & & & & & & & & & & & & & & 10 & 0,57 & 1 & 0,14 & 0,01 & 0,02 & 0,73 \\
\hline 1 & Oenocarpus distichus & & & & & & & & & & & & & & & 10 & 0,57 & 1 & 0,14 & 0,02 & 0,07 & 0,78 \\
\hline 1 & Parinari excelsa & & & & & & & & & & & & & & & 10 & 0,57 & 1 & 0,14 & 0,37 & 1,22 & 1,93 \\
\hline 2478 & TOTAL & 1970 & 100 & 897 & 100 & 34,18 & 100 & 300 & 2260 & 100 & 860 & 100 & 36,56 & 100 & 300 & 1760 & 100 & 721 & 100 & 30,07 & 100 & 300 \\
\hline
\end{tabular}

DAT $=$ Densidade absoluta total ( $\mathrm{N}^{\circ}$ total de indivíduos), $\mathrm{FA}=$ Frequência absoluta (\%), FR = Frequência relativa (\%),

$\mathrm{DA}=$ Densidade absoluta $(\mathrm{n} \% \mathrm{ha}), \mathrm{DR}=$ Densidade relativa $(\%)$,

DoA = Dominância absoluta $\left(\mathrm{m}^{2} / \mathrm{ha}\right)$, DoR = Dominância relativa $(\%), \mathrm{VI}=$ Valor de importância.

DAT $=$ Total Absolute Density $($ Total Individuos number), FA = Absolute Frequence $(\%), F R=$ Relative Frequence $(\%)$,

$\mathrm{DA}=$ Absolute Density $(\mathrm{n} / \mathrm{ha}), \mathrm{DR}=$ Relative Density $(\%)$,

DoA $=$ Absolute Dominance $\left(\mathrm{m}^{2} / \mathrm{ha}\right)$, DoR $=$ Relative Dominance $(\%), \mathrm{VI}=$ Importance Value 
No presente estudo foram encontradas 11 espécies raras, representando $15,9 \%$ do total de espécies identificadas para os 3,0 ha avaliados. Os resultados não coincidiram com os citados por Martins (1991), de trabalhos realizados no Pará e no Amapá. Os resultados foram compatíveis com os de Rabelo (1999), em estudos realizados no Amapá, que encontrou 13 espécies raras no Lontra da Pedreira, representando $19,40 \%$ do total e 19 no Mazagão, representando 14,77\% do total, para 5,0 ha de área estudadas em cada localidade; para as duas áreas encontrou um total de 22 espécies raras, representando $19,29 \%$ do total.

Com relação à diversidade de espécies e a equabilidade (Tabela 3), os resultados foram compatíveis com os encontrados em estudos anteriormente realizados em floresta de várzea, com valores inferiores aos encontrados para terra firme. Segundo Martins (1991), esse resultado deve ser esperado, pois solos que permaneçam por tempo prolongado em condições de drenagem insuficiente, devem restringir o número de espécies que lá podem sobreviver.

Tabela 3. Índice de diversidade de espécies e equabilidade de Shannon-Weaver (H' e J) e quociente de mistura de Jentsch.

Table 3. Diversity species index and equability of Shannon-Weaver ( $\mathrm{H}^{\prime}$ and $\mathrm{J}$ ) and the mixture quotients of Jentsch.

\begin{tabular}{|c|c|c|c|c|c|c|c|c|}
\hline \multirow[t]{2}{*}{ Autor } & \multirow[t]{2}{*}{ Local } & \multirow{2}{*}{$\begin{array}{c}\text { Área } \\
\text { (ha) }\end{array}$} & \multirow{2}{*}{$\begin{array}{l}\text { DAP } \\
(>\mathrm{cm})\end{array}$} & \multirow[t]{2}{*}{ Ambiente } & \multirow[t]{2}{*}{$\mathrm{H}^{\prime}$} & \multirow[t]{2}{*}{$\mathrm{J}$} & \multicolumn{2}{|c|}{ Q. de Mistura } \\
\hline & & & & & & & Total & Dicotiled. \\
\hline Barros & S. M. do Vila Nova/AP & 1,0 & 30 & Terra Firme & 3,58 & - & & \\
\hline Rodrigues & Serra do Navio/AP & 2,6 & 15 & Terra Firme & 3,89 & - & & \\
\hline Black et alii & Belém/PA & 1,0 & 10 & Igapó & 2,63 & - & & \\
\hline Black et alii & Belém/PA & 1,0 & 10 & Terra Firme & 3,72 & - & & \\
\hline Pires et alii & Castanhal/PA & 3,5 & 10 & Terra Firme & 4,30 & - & & \\
\hline Cain et alii & Belém/PA & 2,0 & 10 & Terra Firme & 4,07 & - & & \\
\hline Porto et alii & Manaus/AM & 1,0 & 10 & Mata-de-baixio & 3,59 & - & & \\
\hline Rabelo (1999) & Mazagão/AP & 5,0 & 5 & Várzea & 2,73 & 0,61 & $01: 58$ & $01: 30$ \\
\hline$"$ & Lontra da Pedreira/AP & 5,0 & 5 & Várzea & 1,93 & 0,46 & 01:59 & $01: 24$ \\
\hline$"$ & Mazagão/AP & 1,0 & 5 & Várzea & - & - & 01:20 & $01: 16$ \\
\hline$"$ & Lontra da Pedreira/AP & 1,0 & 5 & Várzea & - & - & $01: 32$ & 01:22 \\
\hline Este trabalho & Rio Maniva/Afuá/PA & 1,0 & 5 & Várzea & 2,84 & 0,75 & $01: 18$ & $01: 12$ \\
\hline$"$ & Mazagão/AP & 1,0 & 5 & Várzea & 2,67 & 0,69 & $01: 18$ & $01: 12$ \\
\hline$"$ & Bailique/AP & 1,0 & 5 & Várzea & 2,31 & 0,65 & $01: 20$ & 01:07 \\
\hline
\end{tabular}

Barros, Rodrigues, Black et alii, Pires et alii, Cain et alii e Porto et alii, citados por MARTINS (1991)

Um dos problemas típicos das florestas tropicais, para o desenvolvimento da atividade de manejo florestal madeireiro, consiste na grande heterogeneidade e na complexa distribuição das espécies, em particular daquelas com valor comercial. Assim, o estudo da distribuição espacial das espécies das florestas da Amazônia representa um dos primeiros passos para o entendimento do estudo integrado das florestas tropicais e para o estudo detalhado de seus componentes (Barros e Machado, 1984).

O entendimento de como as espécies arbóreas se distribuem na área de várzea poderá contribuir para o estabelecimento de projetos de manejo de açaizais, para a coleta de frutos e para a extração de palmito de açaí, com impactos reduzidos sobre o ambiente, mantendo a possibilidade da população do estuário ter assegurado a principal atividade atualmente desenvolvida na área.

De acordo com os índices calculados para as espécies encontradas nos três locais estudados, utilizando-se os procedimentos adotados por Mc Guinnes, observa-se que para a maioria das espécies de destaque, os índices se mostram aplicáveis, mas para um número grande de espécies, não há coincidência nos padrões de distribuição espacial, quando o índice é considerado, variando muito de um local para outro. Mesmo Mora paraensis (pracuúba), espécie de destaque, no furo do Mazagão apresentou padrão de distribuição contagiosa $(6,08)$, no rio Maniva apresentou tendência ao agrupamento $(1,89)$ e no Bailique apresentou distribuição uniforme $(0,39)$ (Tabela 1$)$.

A adoção de metodologias não-padronizadas para a coleta de dados, permite a obtenção de diferentes valores ou índices fitossociológicos para um mesmo ambiente estudado. No caso de açaizeiros, 
há estudiosos que consideram cada estipe como uma planta, outros consideram como uma planta a touceira. A adoção de diferentes perímetros $(\geq 10,15,20,25$ ou $30 \mathrm{~cm})$, o tamanho da amostra e o método de amostragem, podem tornar os resultados divergentes (Tabela 4). Assim, os resultados do presente estudo divergiram dos resultados de alguns estudos realizados em áreas de várzea.

Conforme já citado anteriormente, nas várzeas do estuário do rio Amazonas, a espécie Euterpe oleracea Mart. exibe elevadas densidades. No Bailique, entretanto, a alta densidade de exemplares da família Arecaceae se deve muito mais a ação antrópica. A grande distância entre a Vila Progresso e Macapá inviabilizou a comercialização de frutos de açaí. A extração de palmito se mostrou mais viável e várias fábricas foram instaladas para sua industrialização. Além disso, desde o final da década de 60, o arquipélago vem fornecendo palmito para abastecer indústrias paraenses, localizadas no estuário. A extração de madeira realizada antes e durante a extração do palmito, também contribuiu para o aumento da população dos açaizeiros. Os ribeirinhos do Bailique especializaram-se no fornecimento de palmito, ampliando os açaizais, reduzindo os recursos madeireiros.

No Bailique, em terrenos definidos como projetos de exploração de palmito, a diversidade de espécies é baixa, pois a cada corte de palmito algumas espécies de dicotiledôneas são eliminadas para propiciar o aumento da população dos açaizeiros. O local estudado, pelo fato de pertencer à Escola Bosque do Bailique, há algum tempo não sofre intervenção e, mesmo assim, o número de espécies dicotiledôneas foi mais baixo que nos outros locais.

No rio Maniva, o proprietário da área manteve, até o final da década de 80 , uma pequena serraria e extraía madeira seletivamente. Além disso, extraía palmito nas limpezas do açaizal e para o abastecimento de fábricas de palmito instaladas próximas à área, em regime de baixo impacto. A partir da década seguinte, dedicou-se à coleta de frutos de açaí para comercialização em Macapá e extração seletiva da madeira para construção de casas e confecção de móveis e embarcações de uso familiar. O açaizal foi moderadamente ampliado, com impacto mínimo na população e diversidade das dicotiledôneas.

Tabela 4. Dados de estudos realizados em floresta de várzea da Amazônia.

Table 4. Data of studies realized in Amazon's floodplain forest.

\begin{tabular}{llcccccc}
\hline \multicolumn{1}{c}{ AUTOR } & \multicolumn{1}{c}{ LOCAL } & AMBIENTE & $\begin{array}{c}\text { DAP } \\
(\mathrm{cm})\end{array}$ & $\begin{array}{c}\text { ÁREA } \\
(\mathrm{ha})\end{array}$ & $\begin{array}{c}\text { ESPÉCIES } \\
(\text { Número })\end{array}$ & $\begin{array}{c}\text { DA } \\
(\mathrm{ind} . / \mathrm{ha})\end{array}$ & $\begin{array}{c}\text { DoA } \\
\left(\mathrm{m}^{2} / \mathrm{ha}\right)\end{array}$ \\
\hline Pires e Koury (1954) & Rio Guamá/PA & Várzea & 10 & 3,8 & 107 & 483 & - \\
Mont. e M.-Miret (1997) & Rio Pará/PA & Várzea & 10 & 3,0 & 45 & 477 & 18,87 \\
Rabelo (1999) & Estuário do rio Amazonas & Várzea & 5 & 10,0 & 114 & 1.105 & 36,10 \\
Bentes-Gama (2000) & Afuá/PA & Várzea Alta & 15 & 14,5 & 78 & 289 & 23,70 \\
Jardim e Vieira (2001) & Ilha do Combu/PA & Várzea Alta & 10 & 5,0 & 67 & - & - \\
Este Trabalho & Estuário do rio Amazonas & Várzea Alta & 5 & 3,0 & 69 & 826 & 33,60 \\
\hline
\end{tabular}

DA = Densidade absoluta (n\% ha), DoA = Dominância absoluta $\left(\mathrm{m}^{2} / \mathrm{ha}\right)$,

No Mazagão, o proprietário da área estudada há muito vive da coleta do fruto de açaí e da comercialização da polpa fresca. As intervenções no ambiente natural têm a função principal de limpar o local para facilitar a caminhada na floresta para a coleta dos frutos de açaí. Nos últimos dez anos, priorizou as atividades no açaizal coletando os frutos e extraindo e comercializando a polpa, além de vender o excedente de frutos no mercado de Mazagão. A madeira extraída é utilizada na construção de casas ou barracões na própria área e é feita priorizando o desenvolvimento dos açaizeiros. O açaizal foi moderadamente ampliado, com impacto mínimo na população das dicotiledôneas.

\section{CONCLUSÕES}

A expressão gráfica da curva espécie/área indica que as parcelas foram representativas para os três locais estudados. O número de famílias e espécies encontradas foi inferior ao de outros inventários, em razão das parcelas terem sido alocadas apenas em várzea alta, ecossistema objeto do presente estudo.

A forma de utilização da vegetação no ambiente estuarino tem forte influência na composição florística da área. Em locais onde a coleta de frutos de açaí é a atividade prioritária, as limpezas realizadas nos açaizais para o adensamento populacional dos açaizeiros têm contribuído para a redução da 
diversidade florística. No Bailique, por exemplo, observou-se o menor índice de diversidade entre as áreas estudadas.

A rapidez na regeneração natural, nas áreas de várzea estuarina, após a instalação de um roçado, por exemplo, em razão da boa fertilidade do solo e da alta temperatura local, possibilita o surgimento de ambientes florestais, com os mais diferentes tipos de composições específicas. A rapidez no crescimento das árvores pode levar ao entendimento de que a floresta atingiu seu clímax, ainda que a relação entre espécie e área varie bastante de um local para outro.

\section{AGRADECIMENTOS}

Ao CNPq/FINEP/ PPG7, pelo financiamento parcial deste trabalho.

\section{REFERÊNCIAS}

ANDERSON, A. B.; GELY, A.; STRUDWICK, J.; SOBEL, G. L. \& PINTO, M. C. Um sistema agroflorestal na várzea do estuário amazônico (Ilha das Onças, município de Barcarena, Estado do Pará). Acta Amazônica, Suplemento, v. 15, n. 1/2, p. 195-224. 1985.

BARROS, P. L. C.; MACHADO, S. do A. Aplicação de índices de dispersão em espécies de florestas tropicais da Amazônia brasileira. Curitiba: FUPEF/UFPR, 1984. 44p. (FUPEF - Série Científica n. 1).

BENTES-GAMA, M. M. Estrutura, valoração e opções de manejo sustentado para uma floresta de várzea na Amazônia. Lavras, 2000. 206 f. Dissertação (Mestrado em Engenharia Florestal) Universidade Federal de Lavras.

CURTIS, J. T. The vegetation of Wisconsin. An ordenation of plant communities. Madison: University of Wisconsin Press, 1959.

CURTIS, J. T. \& McINTOSH, R. P. The interrelations of certain analytic and synthetic phytosociological characters. Ecology, v. 31, n. 3, p. 434-50. 1950.

DUBOIS, J. C. L.; VIANA, V. M.; ANDERSON, A. Manual agroflorestal para a Amazônia. Rio de Janeiro: REBRAF, v. 1, p. 93-94. 1996.

DUCKE, A.; BLACK, G. A. Notas sobre a fitogeografia da Amazônia Brasileira. Belém: Instituto Agronômico do Norte, 1954. 62p. (Boletim Técnico n. 29).

JARDIM, M. A. G.; VIEIRA, I. C. G. Composição florística e estrutura de uma floresta de várzea do estuário amazônico, ilha do Combu, Estado do Pará, Brasil. Belém: 2001. p.333-354 (Boletim do Museu Paraense Emílio Goeldi - Série Botânica v. 17, n.2).

LIMA, R. R.; TOURINHO, M. M. Várzeas da Amazônia Brasileira: principais características e possibilidades agropecuárias. Belém: FCAP. Serviço de Documentação e Informação, 1994. 20p.

MARTINS, F. R. Estrutura de uma floresta mesófila. Campinas: Editora da UNICAMP, 1991.

MONTAGNINI, F. \& MUÑIZ-MIRET, N. Vegetacion y suelos de lãs planícies inundables Del estuário Amazónico: una comparación de bosques de "Várzea" y "Terra firme" en Pará, Brasil. Bahia: 1997. p.107-118 (AGROTÓPICA/CEPLAC).

MUELLER-DOMBOIS, D. \& ELLENBERG, H. Aims and methods of vegetation ecology. New York: John Wiley \& Sons, 1974.

NOGUEIRA, O. L. Estrutura e dinâmica populacional de açaizais nativos de várzea na região do Baixo Tocantins, Estado do Pará. Belém: Embrapa Amazônia Oriental, 1999. 21p. (Embrapa Amazônia Oriental. Boletim de Pesquisa n. 15).

PIRES, J. M.; KOURY, H. M. Estudo de um trecho de mata de várzea próximo de Belém. Belém: 1958. p.3-44 (Instituto Agronômico do Norte - Série Boletim Técnico n. 36). 
QUEIROZ, J. A. L. de; MOCHIUTTI, S. Diversidade florestal em sistemas agroflorestais com açaizeiro no estuário amazônico. In: CONGRESSO BRASILEIRO DE SISTEMAS AGROFLORESTAIS, 3., 2000, Manaus. Resumos... São Paulo, 2000. p. 147-149.

QUEIROZ, J. A. L. de; MOCHIUTTI, S. Tipos de açaizais do Estuário Amazônico e efeitos das intervenções praticadas por extratores ribeirinhos. In: SABOGAL, C. \& SILVA, J.N.M. (orgs.) SIMPÓSIO INTERNACIONAL DA IUFRO: Aplicando resultados de pesquisa, envolvendo atores e definindo políticas públicas. Belém, 2002. Anais... CIFOR/Embrapa Amazônia Oriental, 2000. p.344350 .

RABELO, F. G. Composição florística, estrutura e regeneração de ecossistemas florestais na região estuarina do rio Amazonas-Amapá-Brasil. Belém, 1999. 72p. Dissertação (Mestrado em Ciências Florestais) - Faculdade de Ciências Agrárias do Pará.

ZEE/AP. Zoneamento Ecológico Econômico da Área Sul do Estado do Amapá. Macapá: IEPA/GEA/AP, 2000. Atlas. 